\title{
Canagliflozin improves glycemic control and body weight and reduces insulin doses in patients with type 2 diabetes treated with insulin in real practice
}

\author{
Carral $\mathrm{F}^{1 *}$, Cabo $\mathrm{D}^{2}$, Jimenez $\mathrm{AI}^{3}$ and Cayon $\mathrm{M}^{4}$ \\ ${ }^{1}$ Endocrinology and Nutrition Units of Viamed Bahia de Cadiz Hospital, Cadiz, Spain \\ ${ }^{2}$ Quiron Bahia de Algeciras Hospital, Cadiz, Spain \\ ${ }^{3}$ Puerto Real University Hospital, Cadiz, Spain \\ ${ }^{4}$ Jerez University Hospital, Cadiz, Spain
}

\begin{abstract}
Introduction: Canagliflozin is a class of oral anti-diabetic agent with limited real-world experience reported in patients treated with insulin.

Objective: The aim of this study was to evaluate effectiveness and safety of the administration of canagliflozin in patients with type 2 diabetes mellitus (T2DM) treated with different insulin regimens.

Methods: A retrospective observational study of patients with T2DM treated with insulin who were prescribed canagliflozin from June 2015 to December 2016 at four endocrinology clinics in the South of Spain was designed. 12 months changes in anthropometric variables, glycemic control, insulin doses, anti-diabetic drugs and adverse events were assessed after canagliflozin administration.

Results: 92 patients with T2DM treated with insulin (44 women, mean age $61.2 \pm 10.0$ years old and mean BMI: $35.2 \pm 11.2 \mathrm{~kg} / \mathrm{m}^{2}$ ) were studied. Improvement was observed in HbA1c $(8.7 \pm 1.6$ vs. $7.2 \pm 0.8 \%$; p <0.01), body weight $(92.4 \pm 20.9$ vs. $87.1 \pm 19.5 \mathrm{~kg} ; \mathrm{p}<0.01)$ and waist circumference $(112.4 \pm 17.0$ vs. $105.0 \pm 21.7$; p: 0.04$)$, with a reduction of insulin doses $(57.7 \pm 38.9$ vs. $35.1 \pm 32.5 \mathrm{IU} /$ day, $\mathrm{p}<0.01)$ and insulin injections per day $(2.1 \pm 1.2$ vs. $1.1 \pm 0.8, \mathrm{p}<0.01)$ after 12 months of canagliflozin administration. $49 \%$ of the patients reached $\mathrm{HbA} 1 \mathrm{c} \leq 7 \%$ and 12 patients (13\%) completely suspended treatment with insulin. 15 patients $(16.3 \%)$ experienced mild adverse events and canagliflozin administration was suspended in 9 patients (9.8\%).
\end{abstract}

Conclusions: Canagliflozin administration in patients with T2DM treated with insulin was associated with an improvement in glycemic control and body weight and with a reduction of insulin doses with limited and mild adverse events.

\section{Introduction}

Different clinical studies report that less than $50 \%$ of the patients with type 2 diabetes Mellitus (T2DM) treated with insulin achieve adequate metabolic control goals, and at least one-quarter of the patients with T2DM treated with basal insulin have difficulties attaining the recommended $\mathrm{HbAlc}$ goal, despite adequate fasting plasma glucose (FPG) levels [1-4]. In these patients, most of the Clinical Practice Guidelines (CPG) for diabetes $[5,6]$ proposed bolus prandial insulin addition before one or more meals as a more effective strategy than insulin alone, but with important limiting factors such as the develop of hypoglycemia and weight gain. Moreover, weight gain may exacerbate insulin resistance, resulting in an increase of insulin doses need, which may cause further weight gain. However, in recent years CPG propose other alternative strategies for intensifying insulin therapy as the addition of GLP-1 receptor agonist, Dipeptidyl peptidase 4 (DPP-4) inhibitors or Sodium-glucose cotransporter 2 (SGLT2) inhibitors $[7,8]$.

SGLT2 inhibitors offer an anti-diabetic effect increasing urinary glucose excretion and subsequently lowering plasma glucose levels without inducing excessive insulin secretion [9]. Canagliflozin is a SGLT2 inhibitor which has been approved as a complement to diet and exercise for the treatment of adults with T2DM in more than 30 countries, including the United States and the European Union [10]. Therapy combination of canagliflozin and insulin was investigated in a reported sub study of the canagliflozin Cardiovascular Assessment Study (CANVAS) [11] and other few trials [15-19] and it was demonstrated that the addition of canagliflozin to insulin therapy improves glycemic control and decreases weight. However, actually there is little information in real world relating about effectiveness and safety of the addition of canagliflozin to insulin therapy on patients treated in specialty diabetes practical settings [16-19]. Patients in this clinical practice setting usually have longer diabetes disease duration, worst metabolic control and greater complexity medication regimen. While clinical trials results are useful determining clinical outcomes in a representative sample of patients in controlled settings, these results

${ }^{*}$ Correspondence to: Florentino Carral San Laureano, Endocrinology and Nutrition Units of Viamed Bahia de Cadiz Hospital, C/ Arroyuelo 7. 11130 Chiclana (Cadiz). Spain, E-mail: florencarral@hotmail.com

Key words: canagliflozin, obesity, type 2 diabetes mellitus, glycosylated hemoglobin, patients treated with insulin

Received: January 31, 2019; Accepted: February 16, 2019; Published: March 04,2019 

practice

may have limited generalizability when they are compared to realworld administration of canagliflozin with insulin therapies in actual practice. The aim of this study was to evaluate real-world effectiveness and safety of canagliflozin administration in patients with T2DM treated with different insulin regimens.

\section{Patients and methods}

\section{Study design and sample selection}

Retrospective review study of patients with T2DM treated with insulin who were prescribed canagliflozin from June 2015 to December 2016 at four endocrinology clinics in the South of Spain within the context of routine clinical practice. Patients were referred to this specialty consultation room by regional and local specialist and primary care providers for advanced diabetes management, usually because a poor glycemic control. A record search were realized identifying those patients with inclusion criteria: patients with T2DM, over 18 years old, treated with insulin alone or in combination with other anti-diabetic drugs, who started treatment with canagliflozin during the study period and had received initial and regular follow-up in the clinics at least during 12 months. Exclusion criteria were: patients with type 1 diabetes mellitus, pregnant women, patients receiving treatment with systemic corticosteroids during the study period, not having enough information in clinical records or patients who failed two or more programmed visits. Selected patients were included in a database and each one was identified by a unique patient study number assigned to ensure confidentiality.

\section{Outpatient diabetes treatment protocol}

Patients included in the study were attended in one of the four endocrinology outpatient clinics within the context of routine clinical practice. Habitually overweight or obese patients who were on treatment with insulin, in order to promote improvement of metabolic control and weight loss, were instructed in a personalized low-calorie diet and physical activity recommendations. Usually, oral anti-diabetic regimens with sulfonylureas, metiglinides, pioglitazon or acarbose were suspended in every patient and treatment with metformin was maintained or initiated whenever was well tolerated. GLP-1 receptor agonist usually was maintained and initiation was proposed to obese patients. Recommendations were given to patients to optimize basal insulin doses in order to achieve and maintain fasting blood glucose levels between 80 and $130 \mathrm{mg} / \mathrm{dl}$. Insulin doses were reduced when hypoglycemia occurred and insulin therapy discontinuation was proposed to patients who were receiving less than $0.2 \mathrm{IU} \mathrm{kg} /$ day. Prandial insulin doses were adjusted to achieve postprandial glucose below $180 \mathrm{mg} / \mathrm{dl}$ and discontinuation of prandial insulin was indicated when reaching $\leq 6$ IU before meal.

\section{Data collection}

Most of the patients were attended every 3 months, with the possibility of mid-term reviews to assess treatments tolerance and effectiveness. At baseline, demographic variables (gender, age and smoking), history of diabetes (date of diagnosis, current treatment and HbAlc level), comorbidities (hypertension, dyslipidemia) and complications (retinopathy and cardiovascular disease) were collected. In every 3-month visit, body weight, waist circumference (WC), antidiabetic treatment (basal and rapid insulin doses and other anti-diabetic treatments), HbA1c level, common adverse events of SGLT2 inhibitors (mainly osmotic diuresis -polyuria and pollakiuria-, genital candidiasis and urinary tract infection) ketoacidosis and severe hypoglycemia episodes (defined as those episodes requiring help of a third person for resolution) were recorded.

\section{Statistical methodology}

Every data collected in the study was coded, entered, and analyzed using the SPSS Statistics for Windows 12.0 version program. Descriptive analysis of qualitative variables was performed by calculating frequencies and percentages, and for quantitative variables, mean, standard deviation, median, and range was determined. Each patient served as his/her own control. Paired t-tests were performed on primary and secondary outcomes. P values $<0.05$ were considered statistically significant.

\section{IRB approval}

This study was conducted in accordance with the good clinical practice guidelines and with the Helsinki Declaration principles. Study was approved by the independent Institutional Review Board (IRB) of Viamed Hospital.

\section{Results}

92 patients with T2DM (44 female and 48 male) mean age $61.2 \pm$ 10.0 years old, average time with diabetes disease in years $14.8 \pm 9.7$, mean body weight and mean BMI $92.4 \pm 20.9 \mathrm{~kg}$ and $35.2 \pm 11.2 \mathrm{~kg} /$ $\mathrm{m}^{2}$, respectively, were included in the study. Despite using high doses of insulin $(57.7 \pm 38.9 \mathrm{IU}$ daily insulin, $0.6 \pm 0.4 \mathrm{IU} \mathrm{kg} /$ day $)$, patients showed inadequate glycemic control at baseline $(84.8 \%$ of the patients had $\mathrm{HbAlc}>7.0 \%$ ). (Table 1) contains main clinical characteristics of patients included in the study.

After the addition of canagliflozin to the treatment, a significant reduction of $\mathrm{HbAlc}$ was observed from mean baseline value $( \pm \mathrm{SD})$ of $8.7 \pm 1.6 \%$ to $7.2 \pm 0.9 \%$, to $7.1 \pm 0.8 \%$ and to $7.2 \pm 0.8 \%$ on 3,6 and 12 -month visits, respectively (Table 2). At 12 -month visit, total insulin doses, basal insulin and prandial insulin were significantly reduced by an average of $21.1,11.8$ and $10.5 \mathrm{UI} /$ day, respectively, as the number of insulin injections, were reduced by an average of $0.4,0.3$ and 1.0 injections/patient/day, respectively (Table 2). At 12-month visit, $35.8 \%$ (33 patients) of the patients treated with canagliflozin $300 \mathrm{mg}$ had no

Table 1. Clinical characteristics of patients $(n=92)$. Results are expressed as mean \pm standard deviation

\begin{tabular}{|l|c|}
\hline Clinical characteristics & Results \\
\hline Mean Age (years-old) & $61.2 \pm 10.0$ \\
\hline Female (\%) & $44(47.8)$ \\
\hline Mean duration of T2DM (years) & $14.8 \pm 9.7$ \\
\hline Insulin doses (UI/kg/day) & $0.6 \pm 0.4$ \\
\hline Other antidiabetic drugs (\%) & $76(82.6)$ \\
Metformin & $60(65.0)$ \\
Sulfonilureas & $14(15.2)$ \\
DPP-4 inhibitors & $30(32.6)$ \\
\hline GLP-1 receptor agonist & $19(20.7)$ \\
\hline Physical examination & \\
\hline Body weight (kg) & $92.4 \pm 20.9$ \\
\hline BMI (kg/m ${ }^{2}$ ) & $35.2 \pm 11.2$ \\
\hline Waist Circunference (cm) & $112.4 \pm 17.0$ \\
\hline Comorbidities and Chronic complications & \\
\hline Smoking habit (\%) & $16(17.4)$ \\
\hline Dyslipemia (\%) & $66(71.7)$ \\
\hline Hypertension (\%) & $67(72.8)$ \\
\hline Diabetic Retinopathy (\%) & $21(22.8)$ \\
\hline Cardiovascular disease (\%) & $25(27.2)$ \\
\hline
\end{tabular}

BMI: Body mass index; Kg: Kilograms; cm: centimeters; IU: Units 
Carral F (2019) Canagliflozin improves glycemic control and body weight and reduces insulin doses in patients with type 2 diabetes treated with insulin in real practice

Table 2. Clinical and metabolic variables changes at 3,6 and 12 months

\begin{tabular}{|c|c|c|c|c|c|}
\hline & Basal & 3 months & 6 months & 12 months & $\mathbf{P}$ \\
\hline Body weight (Kg) & $92.4 \pm 20.9$ & $89.9 \pm 17.8$ & $86.4 \pm 17.4$ & $87.1 \pm 19.5$ & $<0.01^{1}$ \\
\hline Waist circumference $(\mathrm{cm})$ & $112.4 \pm 17.0$ & $110.2 \pm 14.3$ & $106.6 \pm 13.4$ & $105.0 \pm 21.7$ & $0.13^{2} / 0.01^{3} / 0.04^{4}$ \\
\hline $\mathrm{HbA} 1 \mathrm{c}(\%)$ & $8.7 \pm 1.6$ & $7.2 \pm 0.9$ & $7.1 \pm 0.8$ & $7.2 \pm 0.8$ & $<0.01^{1}$ \\
\hline Patients with $\mathrm{HbA} 1 \mathrm{c} \leq 7 \%(\%)$ & 15.2 & 44.6 & 49.3 & 49.1 & $<0.01^{1}$ \\
\hline Insulin injections/day (n) & $2.1 \pm 1.2$ & $1.7 \pm 1.1$ & $1.8 \pm 1.7$ & $1.1 \pm 0.8$ & $<0.01^{5} / 0.06^{3}$ \\
\hline Patients with rapid insulin (\%) & 42.4 & 34.5 & 27.8 & 15.9 & $<0.01^{1}$ \\
\hline Basal insulin dose (IU) & $42.1 \pm 32.7$ & $37.3 \pm 20.8$ & $33.6 \pm 23.7$ & $28.3 \pm 21.6$ & $<0.01^{1}$ \\
\hline Rapid insulin dose (IU) & $14.7 \pm 24.7$ & $9.4 \pm 18.8$ & $8.5 \pm 18.8$ & $5.4 \pm 16.9$ & $<0.02^{1}$ \\
\hline Total insulin dose (IU) & $57.7 \pm 38.9$ & $45.8 \pm 31.1$ & $41-8 \pm 33.9$ & $35.1 \pm 32.5$ & $<0.01^{1}$ \\
\hline Different types of ADs/day (n) & $1.4 \pm 0.8$ & $2.5 \pm 0.7$ & $2.5 \pm 0.7$ & $2.4 \pm 0.6$ & $<0.01^{1}$ \\
\hline
\end{tabular}

Kg: Kilograms; cm: centimeters; n: number; IU: Units; ADs: Antidiabetic drugs (no insulin), ${ }^{1}$ Three, six and twelve months vs basal; ${ }^{2}$ Three months vs basal; ${ }^{3}$ Six months vs basal; ${ }^{4}$ twelve months vs basal; ${ }^{5}$ Three and twelve months vs basal

differences observed between HbAlc, body weight or insulin doses changes compared with patients who maintained canagliflozin $100 \mathrm{mg}$ from the beginning. 12 patients $(13 \%)$ completely suspended treatment with insulin at 12 months with initial difference between BMI $(30.5 \pm$ 5.6 vs $35.9 \pm 11.9$; p: 0.014$)$, basal insulin initial doses $(20.9 \pm 9$. vs $47.1 \pm 24.5 \mathrm{UI} /$ day; $<0.01)$ and total insulin initial doses $(27.6 \pm 20.1 \mathrm{vs}$ $62.2 \pm 39.1 \mathrm{UI} /$ day; $0.3 \pm 0.2$ vs $0.7 \pm 0.4 \mathrm{UI} / \mathrm{Kg} /$ day; $\mathrm{p}<0.01)$ compared with patients who continued treatment with insulin at 12 months. Additionally, patients treated with canagliflozin lost an average of 5.3 $\mathrm{kg}$ of weight at 12 months, without difference between patients who suspended or not insulin treatment (-5.2 \pm 5.9 vs $-6.4 \pm 9.1 \mathrm{~kg}$; p:0.616) and had reduced their waist circumference an average of $-7.7 \mathrm{~cm}$ at 12 months (p:0.04).

During the follow-up, 15 patients (16.3\%) experienced mild adverse events and canagliflozin administration was suspended for this reason in 9 patients (9.8\%). 7 patients (7.6\%) had genital candidiasis, 3,3\% urinary tract infection and only 1 patient referred important pollakiuria. No patients reported severe hypoglycemia and no ketoacidosis episodes occurred.

\section{Discussion}

The results of our study, conducted within the context of routine clinical practice, confirmed therapy combination of canagliflozin and insulin is associated with a significant reduction in $\mathrm{HbAlc}$, body weight, waist circumference and insulin doses in patients with T2DM majority inadequately controlled, treated with insulin and overweight or obese. Our results provide new information, showing the treatment with canagliflozin improves body weight and glycemic control and was associated with a decrease of insulin injections number, but not to other anti-diabetic agents consumption, which is an additional benefit in terms of reduction in health costs. In this way, in a retrospective observational study conducted in our country with 738 patients with T2DM during 12 months of follow up, it was found patients with a decrease of one point in BMI had lower health spending (especially in pharmacy costs) compared with those patients who increased their BMI during the follow up [20].

Our data are consistent with several clinical trials with canagliflozin [11-14] as well as with others with dapagliflozin [21] and empagliflozin [22] in the sense, the combination of SGLT2 inhibitors and insulin is associated with improvements in plasma glucose levels and body weight, being a real alternative to treatments with basal-bolus $[7,8]$. Specifically, in the CANVAS study combination of insulin and canagliflozin improved HbAlc levels in $-0,58 \%(100 \mathrm{mg})$ and $-0,73 \%(300 \mathrm{mg})$ and body weight in $-2,8 \mathrm{~kg}(100 \mathrm{mg})$ and $-3,5 \mathrm{~kg}(300 \mathrm{mg})$ at 52 weeks [11]. In our study, we observed a greater reduction of HbAlc levels average $-1.5 \%(95 \%$
CI: -0.8 to $-1.8 \%$ ) possibly motivated by inadequate glycemic control of our patients at baseline (HbAlc: $8.7 \pm 1.6$ vs $8.3 \pm 0.9$ in the CANVAS study), similar to other studies with real world data and canagliflozin administration $[17,18]$. However, baseline body weight of our patients was similar to the weight of patients enrolled in the phase III trials with canagliflozin $(92.4 \pm 20.9 \mathrm{Kg}$ vs. $94.8 \pm 22.3 \mathrm{~kg}$ in the CANVAS study), but in our study weight loss average was superior $(-6.2 \mathrm{Kg}$ vs. $-3.5 \mathrm{Kg}$ with canagliflozin 300), maybe related with an increase of use of GLP-1 agonist (40.9\% of patients at 12 month) and an important reduction of insulin doses. In this sense, our patients reduced insulin doses an average of 28.6 and $34.6 \%$ at 6 and 12 months, respectively, and $49 \%$ of the patients reduced more than $20 \%$ of insulin doses at 12 months, much higher decrease than the one observed in phase III trials [1114] or other real world studies with combined therapy of canagliflozin and insulin $[16,17]$. As expected, some patients discontinued treatment before 12-months. Every of these discontinuations were associated with treatment-related adverse events, specifically to genital candidiasis. However, nor serious adverse events occurred.

The present study has several limitations. First, it is a multicenter study with a small cohort of patients with T2DM, overweight or obese, evaluated for a short period of time (12 months) without a control group associated. Second, despite the availability of treatment protocol and uniform monitoring, it is possible in some cases it could has not been strictly followed. Third, no patient received treatment with orlistat or other anti-obesity medications, which could have contributed to further improve metabolic control and body weight of our patients. Finally, does not assess the impact on other important clinical parameters such as lipid profile or blood pressure levels, among others.

\section{Conclusion}

In conclusion, our data support the idea that canagliflozin addition to the treatment of inadequately controlled, overweight or obese, patients with T2DM treated with insulin, is associated with an improvement in glycemic control and body weight and with a reduction of the number of insulin doses and injections. There were limited and mild adverse events, although few led to treatment discontinuation.

\section{Funding}

No funding or sponsorship was received for this study or publication of this article.

\section{Disclosures}

Authors declare they have no conflict of interest. 


\section{Compliance with ethics guidelines}

All procedures followed were in accordance with the ethical standards of the responsible committee on human experimentation (institutional and national) and with the Helsinki Declaration of 1964, as revised in 2013.

\section{References}

1. Perez A, Mediavilla JJ, Minambres I, Gonzalez-Segura D (2014) Glycemic control in patients with type 2 diabetes mellitus in Spain. Rev Clin Esp 214: 429-436. [Crossref]

2. Mata-Cases M, Mauricio D (2017) Clinical characteristics of type 2 diabetic patients on basal insulin therapy with adequate fasting glucose control who do not achieve $\mathrm{HbAlc}$ targets. J Diabetes 9: 34-44. [Crossref]

3. Heinjes EM, Thomsen TL, Penning-Van Beest FJ, Christensen TE, Herings RM (2010) Glycemic control and long-acting insulin analog utilization in patients with type 2 diabetes. Adv Ther 27: 211-222.

4. Liebl A, Jones S, Goday A, Benroubi M, Castell C, et al. (2012) Clinical outcomes after insulin initiation in patients with type 2 diabetes: 24-month results from INSTIGATE. Diabetes Ther 3: 9. [Crossref]

5. Menendez Torre E, Lafita Tejedor J, Artola Menendez S, Millan Nunez-Cortes J, Alonso Garcia A, et al. (2011) [Recommendations for the pharmacological treatment of hyperglycemia in type 2 diabetes]. Rev Clin Esp 211: 147-155. [Crossref]

6. Inzucchi SE, Bergenstal RM, Buse JB, Diamant M, Ferrannini E, et al. (2015) Management of hyperglycemia in type 2 diabetes, 2015: a patient-centred approach update to a position statement of the american diabetes association and the European association for the study of diabetes. Diabetes Care 38: 140-149. [Crossref]

7. Artola S, Mata Cases M, Ezkurra P, Navarro J, Martín E (2017) Consenso para la insulinización en diabetes mellitus tipo 2 de la RedGDPS. Diabetes Practica 8: 1-24.

8. Garbe AJ, Abrahamson MJ, Barzilay JI, Blonde L, Bloomgarden ZT, et al. (2017) Consensus statement by the American association of clinical endocrinologists and American college of endocrinology of the comprehensive type 2 diabetes management algorithm- 2017 Executive summary. Endocr Pract 23: 207-238.

9. Bailey CJ (2011) Renal glucose reabsorption inhibitors to treat diabetes. Trends Pharmacol Sci 32: 63-71.

10. Deeks ED, Scheen AJ (2017) Canagliflozin: A review in type 2 diabetes. Drugs 77: 1577-1592. [Crossref]

11. Neal B, Perkovic V, de Zeeuw D, Mahaffey KW, Fulcher G, et al. [2015] Efficacy and safety of canagliflozin, an inhibitor of sodium-glucose cotransporter 2, when used in conjunction with insulin therapy in patients with type 2 diabetes. Diabetes Care 38: 403-411.
12. Van Gaal L, Dumas R, Matthews DR, Desai M, Capuano G, et al. (2015) Efficacy and safety of canagliflozin added to background insulin in patients with type 2 diabetes mellitus in subgroups by baseline insulin dose. Poster presented at the $51 \mathrm{st}$ annual meeting of the European association for the study of diabetes (EASD); 14-18 September 2015; Stockholm, Sweden.

13. Inagaki N, Harashima S, Maruyama N, Kawaguchi Y, Goda M, et al. (2016) Efficacy and safety of canagliflozin in combination with insulin: a double-blind, randomized, placebo-controlled study in Japanese patients with type 2 diabetes mellitus. Cardiovasc Diabetol 15: 89. [Crossref]

14. Inagaki N, Harashima S, Kabu K, Kondo K, Maruyama N, et al. (2017) Long-term efficacy and safety of canagliflozin in combination with insulin in Japanese patients with type 2 diabetes mellitus. Diabetes Obs Metab 20: 812-820. [Crossref]

15. Matsumura M, Nakatani Y, Tanka S, Aoki C, Sagara M, et al. (2017) Efficacy of additional canagliflozin administration to type 2 diabetes patients receiving insulin therapy: examination of diurnal glycemic patterns using continuous glucose monitoring (CGM) Diabetes Ther 8: 821-827. [Crossref]

16. Nardolillo AM, Kane MP, Busch RS, Watsky J, Hamilton R (2014) A clinical perspective of canagliflozin in the management of type 2 diabetes mellitus. Clinical medicine insights: endocrinology and diabetes 7: 25-30.

17. Johnson JF, Parsa R, Bailey R (2017) Real world clinical outcomes and patient characteristics for canagliflozin treated patients in a specialty diabetes clinic. Curr Med Res Opin 33: 77-84. [Crossref]

18. Bailey RA, Schwab P, Xu Y, Pasquale M, Renda A (2016) Glycemic control outcomes after canagliflozin initiation: observations in a medicare and commercial managed care population in clinical practice. Clinical Therapeutics 38: 2046-2057.

19. Buysman EK, Anderson A, Bacchus S, Ingham M (2017) Retrospective study on the impact of adherence in achieving glycemic goals in type 2 diabetes mellitus patients receiving canagliflozin. Adv Ther 34: 937-953. [Crossref]

20. Dilla T, Valladares A, Nicolay C, Salvador J, Reviriego J, et al. (2012) Healthcare costs associated with change in body mass index in patients with type 2 diabetes mellitus in spain. The ECOBIM study. Appl Health Econ Health Policy 10: 417-430.

21. Wilding JP, Woo V, Rohwedder K, Sugg J, Parikh S, et al. (2014) Dapagliflozin in patients with type 2 diabetes receiving high doses of insulin: efficacy and safety over 2 years. Diabetes Obes Metab 16: 124-136. [Crossref]

22. Rosenstock J, Jelaska A, Frappin G, Salsali A, Kim G, et al. (2014) Improved glucose control with weight loss, lower insulin doses, and no increased hypoglycemia with empagliflozin added to titrated multiple daily injections of insulin in obese inadequately controlled type 2 diabetes. Diabetes Care 37: 1815-1823. [Crossref]

Copyright: C2019 Carral F. This is an open-access article distributed under the terms of the Creative Commons Attribution License, which permits unrestricted use, distribution, and reproduction in any medium, provided the original author and source are credited. 